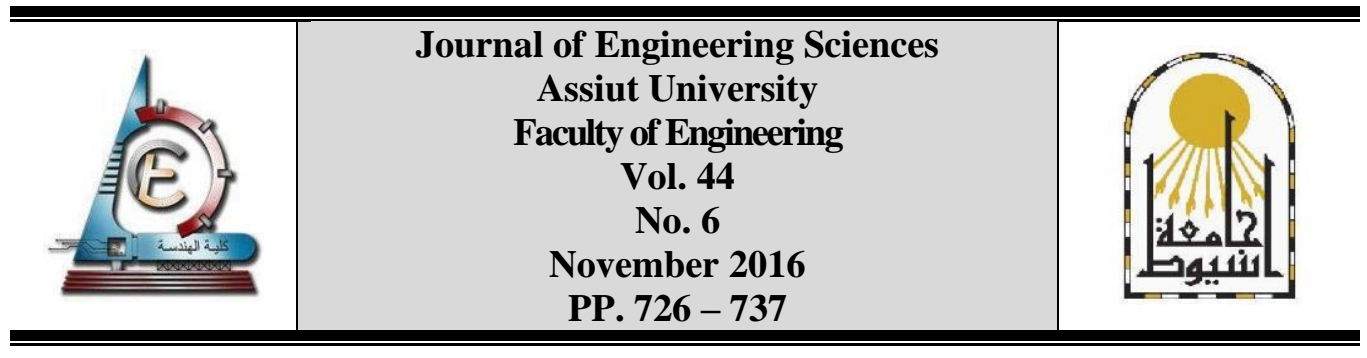

\title{
ENHANCED SATELLITE IMAGES FUSION ALGORITHM USING COMBINED BI-DIMENSIONAL EMPIRICAL MODE DECOMPOSITION AND SHEARLET TRANSFORM BASED ON QGA OPTIMIZATION
}

\author{
Usama Sayed Mohamed ${ }^{1}$, Osama Ahmed Omer ${ }^{2,3}$, and Amal Ahmed Hamed ${ }^{4}$ \\ ${ }^{1}$ Electrical Engineering Department, Assiut University, Assiut, Egypt \\ ${ }^{2}$ Electrical Engineering Department, Aswan University, 81542, Aswan, Egypt \\ ${ }^{3}$ Arab Academy for Science, Technology and Maritime Transport, Aswan, Egypt \\ ${ }^{4}$ National Authorities for Remote Sensing and Space Science, Aswan, Egypt
}

Received 21 August 2016; Accepted 28 September 2016

\begin{abstract}
The satellite images fusion is a process of merging data of the same scene from the panchromatic (PAN) and multispectral (MS) images captured by different instruments. This paper introduces a new satellite images fusion method using Bidimensional Empirical Mode Decomposition (BEMD) and shearlet Transform (ST). BEMD is an efficient method for satellite images processing due to its advantage of spectral data maintaining. Basically, it is used to transform the MS image into subsets named Intrinsic Mode Functions (IMFs). Hence these IMFs and the PAN image are divided by shearlet into high and low frequencies subsets, then the high frequency ones of IMFs are replaced with their corresponding subsets of the PAN image. At last, inverse shearlet and inverse BEMD are implemented to get the fused MS image. Shearlet is preferred due to its optimal representation of the anisotropic elements in the image and due to its capability in processing the continuity and digital data unlike curvelet and contourlet. The experiment results illustrated that the proposed method extracts more spatial details from PAN images with fewer losses in spectral quality of MS images compared to other classic fusion methods. For more enhancements, the fusion weights are estimated efficiently by a quantum genetic algorithm (QGA)-based approach.
\end{abstract}

Keywords: Image, satellite, fusion, BEMD, Shearlet, QGA.

\section{Introduction}

Satellite images fusion is a process of combining a low spatial-resolution MS image with a high spatial-resolution PAN image into a higher resolution MS image. It can show more details and information than observed in individual images. So image fusion technique is important for many remote sensing applications. The most commonly fusion methods in remote sensing are Intensity Hue Saturation (IHS), multiplicative, Principal Component Analysis (PCA) and Brovey transform (BT). However, the main disadvantage of these methods is color "spectral" distortion in the resulted fused MS image [1]. Other methods

Corresponding author.

Email address: Usama@aun.edu.eg 
such as discrete wavelet transforms (DWT) maintain more spectral information but lose spatial details in the output images. Although wavelet is good in representing images with sharp features, but it cannot deal with folded edges have multi directions such as curves, because it is isotropic method created by extending set of objects [2]. It is obvious that in images which contain contours and curves, wavelet cannot process efficiently these anisotropic objects that being observed by place and trend. Over the previous years, several multiscale geometric analysis (MGA) tools such as contourlet and curvelet [3] have been proposed to treat with multidimensional images. Due to their directions sensitivity, they are suitable to represent the precise geometrical features in satellite images. In this context, shearlet as a new MGA tool was proposed by Labate in 2005 [4]. It is actually the only method that provides qualified sparse representations of digital images and also permits for a unified processing in the continuity and digital data [5]. For that, shearlet can be used to overcome most drawbacks of wavelet, curvelet and contourlet.

In the frequency domain, empirical mode decomposition (EMD) is introduced in 1998 by Huang [6] as a signal processing method to handle nonlinear data. Nunes [7] extended EMD to bi-dimensional EMD (BEMD) to represent the image details and texture. It also has been examined in the field of images fusion [8]. EMD has been compared with wavelet for denoising, processing, and classification in [9] to prove its efficiency. In 2012, a rabid and adaptive BEMD for image fusion is developed by Ahmed [10]. In 2014, Weihua [11] proposed a new approach using BEMD for merging the MS and PAN images. Because of all these previous studies, many researchers in the field of remote sensing images fusion were interested in the BEMD. The main advantage of BEMD is its spectral data preserving in the output fused MS images while the main drawback of other classic fusion methods such as IHS, multiplicative and Brovey is their spectral color distortion.

Quantum genetic algorithm (QGA) is an integration of quantum computing and genetic algorithms, and it is in fact an exploration method of probability. It is introduced first by Narayanan in 1996 [12]. QGA outperforms the classic GA by its high divergence, rabid convergence and wide global search [13]. It is a powerful method for solving the optimization equations based on a natural selection operation; it can be used in image processing methods for choosing optimal parameters and setting.

This paper is organized as follows. Section 2 presents standalone image fusion methods; BEMD and the shearlets and presents the QGA. Section 3 describes the proposed integrated scheme BEMD+Shearlet. We enhance the proposed approach by implementing automatic adaptive QGA optimization technique to select the fusion weights in section 4. Section 5 shows the experimental results and the comparison with other methods used in this field. Finally, conclusion is explained in Section 6.

\section{Images fusion methods in remote sensing}

\subsection{BEMD}

This method does not need a priori information and it starts from the image contents for decomposing into a finite number of intrinsic mode functions (IMFs) and residue. These decomposed parts can be reconstructed into an image again after operations; because basically these intrinsic functions in addition to the residue give the original image. The first IMF is considered a high pass filtered image; which includes the highest frequencies. The remaining IMFs include band pass filtered image till low frequencies. BEMD process is modeled by Equation: 
JES, Assiut University, Faculty of Engineering, Vol. 44, No. 6, November 2016, pp. 727 - 737

$$
X(t)=\sum_{i=1}^{n} I M F_{i}+R_{n}
$$

Where $\mathrm{X}(\mathrm{t})$ is the original image and $\mathrm{R}_{\mathrm{n}}$ is the residue. The Bi-dimensional sifting process is explained as follows:

\section{The Bi-dimensional sifting process:}

1. Initialization: $\mathrm{C}_{0}=\mathrm{r}$ (residue) and $\mathrm{k}=1$ (IMF No.).

2. Obtaining the $\mathrm{k}^{\text {th }} \mathrm{IMF}: \mathrm{I}_{\mathrm{k}}(\mathrm{m}, \mathrm{n})$

a) generate a variable $E_{0}(m, n)$ to equal $r_{k-1}(m, n)$ which is the residual data and $j$ (IMF No.) equal " 1 "

b) Detect all minima and maximum of $\mathrm{E}_{\mathrm{j}-1}(\mathrm{~m}, \mathrm{n})$ in rows and columns.

c) Apply surface interpolation (spline function) by using the minima and maximum to obtain 2-D upper envelopes " $\operatorname{Envmin}_{\mathrm{j}-1}(\mathrm{~m}, \mathrm{n})$ ") and lower envelopes "Envmax $\mathrm{j}_{\mathrm{j}-1}(\mathrm{~m}, \mathrm{n})$ ",

d) Calculate the mean value of the two envelopes:

$$
m_{j-1}(m, n)=\left(\operatorname{Envmin}_{j-1}(m, n)+\operatorname{Envmax}_{j-1}(m, n)\right) / 2
$$

e) Update intermediate variable $\mathrm{Ej}-1(\mathrm{~m}, \mathrm{n})$ by subtracting the average envelope from the original image $\mathrm{EO}(\mathrm{m}, \mathrm{n})$ to obtain $\mathrm{Ej}(\mathrm{m}, \mathrm{n})$ which represents the jth iteration in ith sifting operation.

$$
E_{j}(m, n)=E_{j-1}(m, n)-m_{j-1}(m, n)
$$

f) Examine the stopping condition; if it is "yes", the sifting is stopped and go to 3, and then we can get the IMFi using Equation (4). If not, place $\mathrm{j}=\mathrm{j}+1$ and go through steps $a-f$

$$
I_{k}(m, n)=E_{j}(m, n)
$$

3. Replace the residue as equation (5), repeat steps $1-3$ with $k=k+1$ till the number of extrema in the residue image are less than 2 or have no change. Then, the BEMD sifting operation is finished [14].

$$
\mathrm{r}_{k}(\mathrm{~m}, \mathrm{n})=\mathrm{r}_{k-1}(\mathrm{~m}, \mathrm{n})-I_{k}(m, n)
$$

The sum of all parts and the residual one are added to reconstruct the original image in the following equation [15]:

$$
C(m, n)=\sum_{k=1}^{K} I_{k}(m, n)+r(m, n), k \in N^{*}
$$

\subsection{Shearlet}

The shearlet transform is actually based on the classical theory of affine structures. In case of two-dimension data, the affine system with complicated dilations is modeled as following [16]:

$$
\psi_{A B}\left(\psi_{A B}\right)=\left\{\psi_{j, l, k}(x)=|\operatorname{det} A|^{j / 2} \psi\left(B^{l} A^{j} x-k\right): j, l \in z, k \in z^{2}\right\}
$$

Where $\psi \in \mathrm{L}^{2}\left(\mathrm{R}^{2}\right)$, A and B are $2 \times 2$ invertible matrices. The frame $f$ should satisfy the following:

$$
\begin{gathered}
\sum_{j, l, k}\left|\left\langle f, \psi_{j, l, k}\right\rangle\right|^{2}=\|f\|^{2}, \forall f \in L^{2}\left(R^{2}\right) \\
A=\left[\begin{array}{cc}
a & 0 \\
0 & \sqrt{a}
\end{array}\right](a>0), B=\left[\begin{array}{ll}
1 & s \\
0 & 1
\end{array}\right](s \in R)
\end{gathered}
$$


The shearlet is an example of these affine systems, where its elements are composite wavelets with several scales. The anisotropic dilation matrix A represents the scale transform and the shear matrix B related with place preserving geometric transformation such as rotations and oblique shapes; it maintains the digital structure [17]. According to the definition of continuous shearlet transform

$$
\begin{gathered}
\psi_{a . s, t}(x)=a^{-3 / 4} \psi\left(S_{s}^{-1} A_{a}^{-1}(x-t)\right) \\
A_{a}=\left(\begin{array}{cc}
a & 0 \\
0 & \sqrt{a}
\end{array}\right), S_{s}=\left(\begin{array}{ll}
1 & s \\
0 & 1
\end{array}\right), \psi \in L^{2}\left(R^{2}\right)
\end{gathered}
$$

It follows these conditions:

- $\hat{\psi}(\varepsilon)=\hat{\psi}\left(\varepsilon_{1}, \varepsilon_{2}\right)=\hat{\psi}_{1}\left(\varepsilon_{1}\right) \hat{\psi}_{2}\left(\varepsilon_{2} / \varepsilon_{1}\right)$

- $\hat{\psi}_{1}(\varepsilon)=C^{\infty}(R)$ supp $\psi_{1} \subset\left[-2,-\frac{1}{2}\right] \cup\left[\frac{1}{2}, 2\right]$, where $\psi_{1}$ is a continuous wavelet

- $\hat{\psi}_{2}(\varepsilon)=C^{\infty}(R) \operatorname{supp} \hat{\psi}_{2} \subset[-1,1]$, where $\hat{\psi}_{2}>0$ but $\left\|\psi_{2}\right\|=1$

For $\psi_{a, s, t}, \mathrm{a} \in \mathrm{R}^{+}, \mathrm{r}$ and $\mathrm{t} \in \mathrm{R}^{+}$, for any $f \in \mathrm{L}^{2}\left(\mathrm{R}^{2}\right)$

Where a, s, trepresent the scale conversions, the shear direction and the translation value, respectively [18]. Discrete shearlet transform performs multiscale and direction operations individually.

As shown in Fig.1, at first Laplace pyramid is used to decompose $\mathrm{f}_{\mathrm{a}}^{\mathrm{j}-1}$ into low pass image $f_{a}^{j}$ and a high pass image $f_{b}{ }^{j}$ with $N_{j}=N_{j-1} / 4$; where $j$ is the resolution level. Second step; the shearlet direction filter is performed; the estimated ${ }^{\wedge} \mathrm{f}_{b}^{j}$ is computed on the pseudo-polar grid by one-dimensional band-pass filter, which gives a matrix $\mathrm{D}^{\wedge} \mathrm{f}_{\mathrm{b}}^{\mathrm{j}}$. Another band-pass filter is used on matrix $\mathrm{D}^{\wedge} \mathrm{f}_{\mathrm{b}}{ }^{\mathrm{j}}$ to compute the sampled values. Different subsections and different directions shearlet coefficients are got in this step [17]. At last the reconstructed image is estimated by using the inverse two-dimensional Fast Fourier Transform (FFT). The main drawback of shearlet is the processing speed; it is slower than the discrete wavelet transform, however remains in the range of the processing time of other directional transforms.

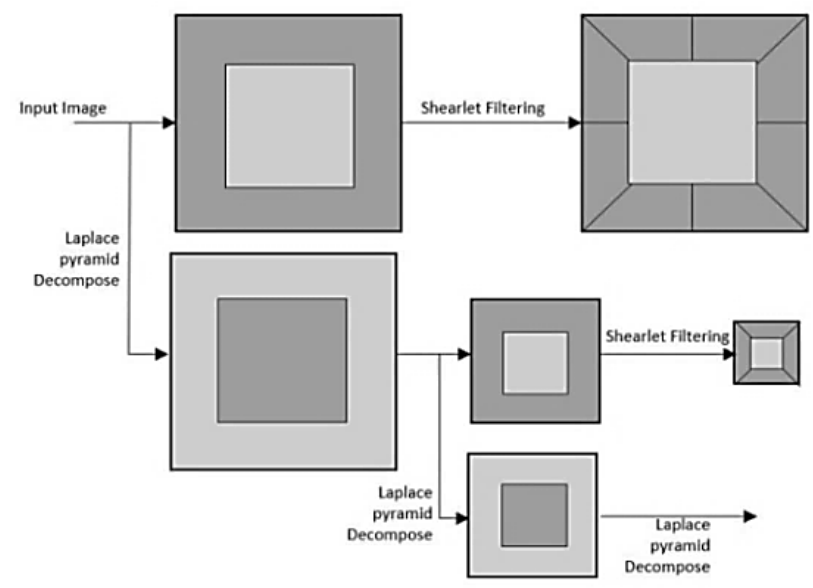

Fig. 1. Laplace pyramid and direction filter "shearlet transform" [18] 
JES, Assiut University, Faculty of Engineering, Vol. 44, No. 6, November 2016, pp. 727 - 737

\subsection{Quantum genetic algorithm}

The smallest unit (bit) of the digital binary data in classic computing is represented by 0 or 1. However, the quantum computing uses what is called qubit; it can be in the "1" state, in "0" state or in any superposition of the two states [19]. A state of a qubit is described as follows:

$$
|\Psi>=\propto| 0>+\beta \mid 1>
$$

Where $\mid 0>$ and $\mid 1>$ represent respectively the classical bit values 0 and $1, \alpha$ and $\beta$ are complex variables satisfy the following condition:

$$
|\alpha|^{2}+|\beta|^{2}=1
$$

$|\alpha|^{2}$ is the probability to get the value 0 and $|\beta|^{2}$ is the probability to get the value 1 . However, when the 'measure' or 'observe' is done, the qubit will equal the value of a single state. In case of m-qubits data, the resulting state space has $2^{\mathrm{m}}$ dimensions. This could lead to exponentially faster converging than the classical computers. The QGA chromosome is a set of $\mathrm{N}$ qubits as the following:

$$
q_{j}^{t}=\left[\begin{array}{llll}
\alpha_{11}^{t} \alpha_{12}^{t} . . \alpha_{1 k}^{t} & \ldots \ldots . . & \alpha_{m 1}^{t} \alpha_{m 2}^{t} . . \alpha_{m k}^{t} \\
\beta_{11}^{t} \beta_{12}^{t} . . \beta_{1 k}^{t} \ldots \ldots . . & \beta_{m 1}^{t} \beta_{m 2}^{t} . . \beta_{m k}^{t}
\end{array}\right]
$$

Where $\mathrm{q}_{\mathrm{j}}^{\mathrm{t}}$ represents the $\mathrm{j}^{\text {th }}$ chromosome of the $\mathrm{t}^{\text {th }}$ generation, $\mathrm{k}$ represents the No. of qubit in individual gene, $\mathrm{m}$ represents No. of genes in each chromosome. Comparing with the traditional GA that uses crossover and mutation to achieve population divergence, in QGA the chromosome values are updated by a $\mathrm{Q}$ gate as the following:

$$
\left[\begin{array}{l}
\alpha_{i}^{\prime} \\
\beta_{i}^{\prime}
\end{array}\right]=U\left(\Delta \theta_{i}\right)\left[\begin{array}{l}
\alpha_{i} \\
\beta_{i}
\end{array}\right]=\left[\begin{array}{cc}
\cos \left(\Delta \theta_{i}\right) & -\sin \left(\Delta \theta_{i}\right) \\
\sin \left(\Delta \theta_{i}\right) & \cos \left(\Delta \theta_{i}\right)
\end{array}\right]\left[\begin{array}{l}
\alpha_{i} \\
\beta_{i}
\end{array}\right]
$$

Where $\alpha_{\mathrm{i}}$ and $\beta_{\mathrm{i}}$ represent the qubit before replacement and $\alpha_{\mathrm{i}}^{\prime}$ and $\beta_{\mathrm{i}}^{\prime}$ represent it after replacement. $\Delta \theta_{\mathrm{i}}$ represents the rotation angle; its lookup table is explained in [20]. Therefore, the main feature of QGA is its wide search capability as well as converging speed. However, this efficient performance is not affected with the small population size [21].

\section{The proposed combined BEMD+shearlet fusion algorithm}

In order to improve spatial resolution of fused images while maintaining spectral quality as high as possible, we propose a new modification of the BEMD fusion by utilizing shearlet transform that varies the manner the high spatial details is injected into the output fused image. Shearlet is able to capture the sharp geometrical elements in remote sensing images due to its accurate directional representation advantages while wavelets can detect only limited directional objects.

To minimize spectral distortion the IMF component should approximate the substituted PAN image as closely as possible. The high spatial details and the spectral information are merged by adaptive weights obtained automatically by QGA-based optimization technique according to image itself and the required sharpness as appeared to user. Therefore, in this integrated BEMD + Shearlet fusion method we want to determine the weights that achieve best sharpness with less spectral distortion.

The flowchart of the proposed method is presented in Fig. 2. Corresponding texts are described as shown in Algorithm 1. 


\section{Algorithm 1: Proposed BEMD+Shearlet method}

Input: PAN and MS image

1. Up-sample MS image to same size as PAN image.

2. The up-sampled MS image is BEMD-decomposed obtaining the IMF and residue.

3. Match the histogram of PAN image to histogram of the IMF component to produce new matched PAN image.

4. Decompose IMF component and new matched PAN images into low-frequency coefficients and high-frequency coefficients by shearlet transform.

5. Fuse the low and high frequency coefficients according to QGA-Based fusion rule

6. Obtain the fused IMF component from the fused low and high frequency coefficients by the inverse Shearlet transform.

7. Reconstruct the fused MS image by adding the new IMF and original residue (inverse BEMD).

Output: fused sharpened MS image.

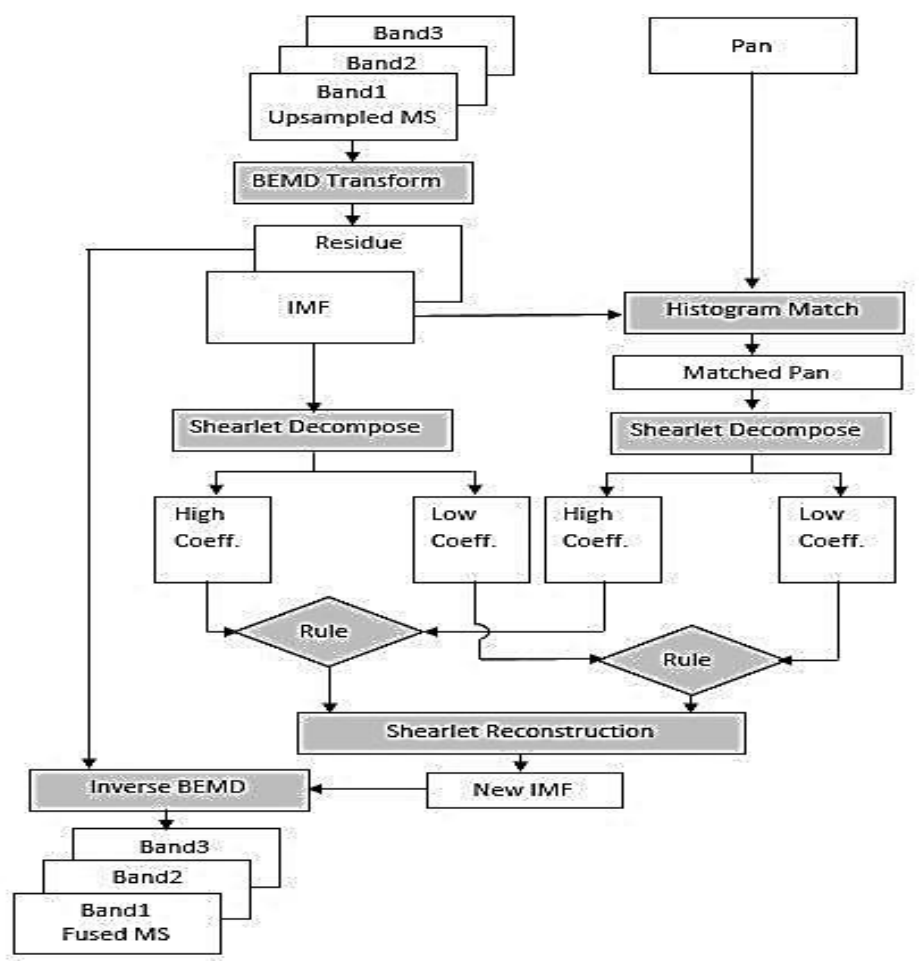

Fig. 2. The flow diagram of proposed BEMD+Shearlet algorithm

\section{Quantum genetic-based BEMD+shearlet image fusion enhancement}

The main goal of using QGA is to improve and adaptive the performance of the image fusion by choosing optimal or quasi-optimal fusion weights automatically instead of assuming or try and error.

\section{The overall structure of QGA}

1. Initialize the population $\mathrm{Q}\left(\mathrm{t}_{0}\right)$; the initial $(\alpha, \beta)$ of each individual are selected to be equal $1 / \operatorname{sqrt}(2)$ [22], that to begin presenting all states with equal probability. 
JES, Assiut University, Faculty of Engineering, Vol. 44, No. 6, November 2016, pp. 727 - 737

2. Produce $\mathrm{P}(\mathrm{t})$ by observing states of $\mathrm{Q}(\mathrm{t})$ (extract a classic gene from a quantum gene selected randomly based on the $\alpha$ and $\beta$ values of the qubit).

3. Evaluate the fitness of every solution of $\mathrm{P}(\mathrm{t})$ by applying fitness function (objective function).

4. Use the best evaluated solution in next level as the new updated one.

5. Update population $\mathrm{Q}(\mathrm{t})$ by quantum rotating gate, that obtain $\mathrm{Q}(\mathrm{t}+1)$.

6. Store the best solution and its fitness value.

7. Repeat above steps till convergence to an optimum value or till no improving can be get.

\section{Experimental results and analysis}

The proposed scheme is evaluated using two sets of different satellites images; one is for Worldview-3 satellite, the MS image has $1.24 \mathrm{~m}$ resolution and the PAN image has $31 \mathrm{~cm}$ resolution, the other set is for Quickbird Satellite, the MS image has $2.16 \mathrm{~m}$ resolution and the PAN image has $55 \mathrm{~cm}$ resolution, as shown in Figs. 3 and 5 in respectively. The images are selected to include different features (urban, vegetation, roads, rocks and seas) to visually evaluate the color spectral distortions over most images features.

In the proposed method, BEMD is used in integration with one of multiresolution analysis (MRA) tools; the shearlet transform for improving the fused image quality as described in previous section. In BEMD image decomposition, the IMF is the extracted highest-frequency component, and the residual is the low-frequency one. The stop criteria limit is selected to be 2; most low frequency component information is extracted at first sifting time. By experiments, we noticed that increasing sifting time reduces the algorithm efficiency. In shearlet decomposition, directions are selected to be $(1,8,8,16$, and 16) in the scales from coarser to finer, respectively.

For enhancement, QGA is used to adaptively select the fusion weights (fusion rule); it is implemented based on required standard deviation of fused pixel values. For fairness, histogram matching is employed before fusion step to ensure consistency between the PAN image and substituted IMF components. For comparison, we tried other MRA tools such as wavelet and curvelet. Figs. 4 and 6 show a comparison of the output fused images obtained using the proposed BEMD+Shearlet, BEMD+Curvelet, and BEMD+Wavelet methods. By visual inspection, the new BEMD+Shearlet method has higher spatial details compared to other methods while maintains spectral quality and shows natural colors. The BEMD+Wavelet method has reasonable spectral quality but it has less spatial improvement.

As it cannot easily be determined which method is better in terms of the clarity of the fused images visually, the quantitative metrics are used, as shown in tables 1 and 2, metrics such as Mean Square Error (MSE), Correlation Coefficient (Cor.) and Quality Without Reference (QNR) [23] are employed to quantitatively analysis the spatial (spat.) quality of fused images, They are calculated between output fused image and input PAN image except QNR; which is a global metric, it does not require a reference. For spectral (spec.) evaluation, Mean Structural Similarity (MSSIM) Index is calculated between output fused image and input MS image by comparing their illuminance, contrast, and structure [24]. Peak Signal to Noise Ratio (PSNR) [23] in decibel (DB) is calculated between output fused images and both inputs MS and PAN images to evaluate the spectral and spatial quality in respectively and then averaged values of both evaluations are taken to show the spatial enhancement while maintaining the spectral quality, the same for the metric Relative Dimensionless Global Error in Synthesis (ERGAS) [23] as shown in tables 1 and 2. In addition, Universal Image Quality Index (UIQI) metric is used as a global metric for evaluating whole image quality; it shows the degradation as a combination of three variables: correlation loss, illuminance and contrast decrease [23]. 
733

Amal Ahmed Hamed et al., Enhanced satellite images fusion algorithm using combined ...........

Based on the results mentioned in tables 1 and 2 and plots in figs 7 and 8, it may be concluded that: BEMD+Wavelet keep the spectral information but result in lower spatial quality. The overall ability of proposed BEMD+Shearlet is better than the other methods, its performance is good in sense of MSE, Cor. and QNR but less efficient in sense of MSSIM (spectral distortion), and BEMD+Wavelet method performance is good in spectral assessment (MSSIM) but bad in sense of MSE, Cor. and QNR. The proposed BEMD+Shearlet method is better than other methods in keeping spectral information while enhancing the spatial details, in terms of PSNR, ERGAS and UIQI.

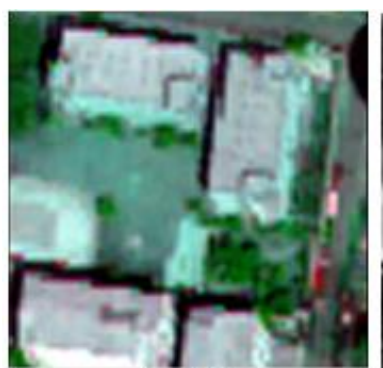

a) MS

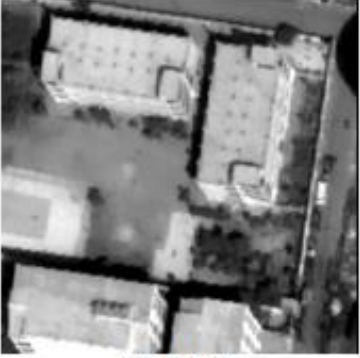

b) PAN

Fig. 3. Input WorldView3 satellite image

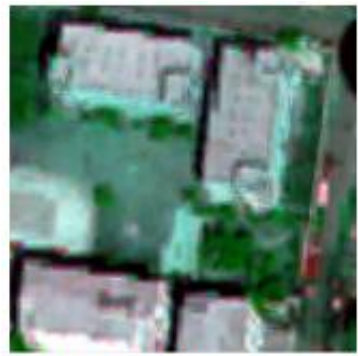

a) BEMD+Wavelet

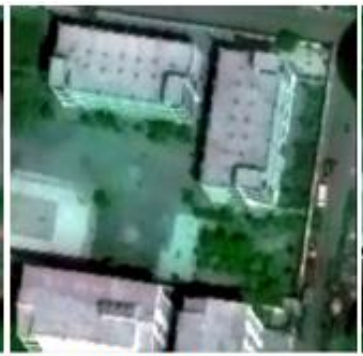

b) BEMD+Curvelet

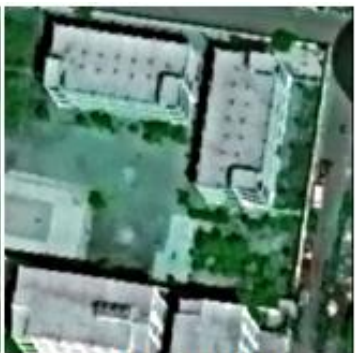

c) proposed BEMD+Shearlet

Fig. 4. Comparison of different fusion methods by using WorldView3 images

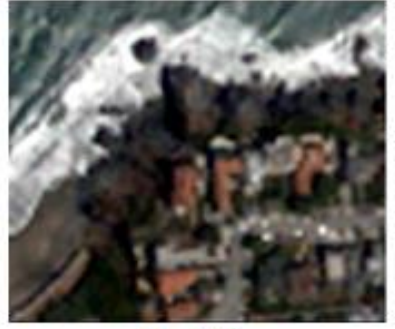

a) MS

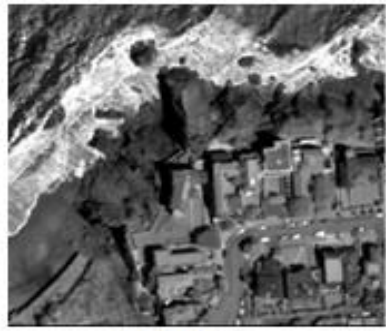

b) PAN

Fig. 5. Input Quickbird satellite images
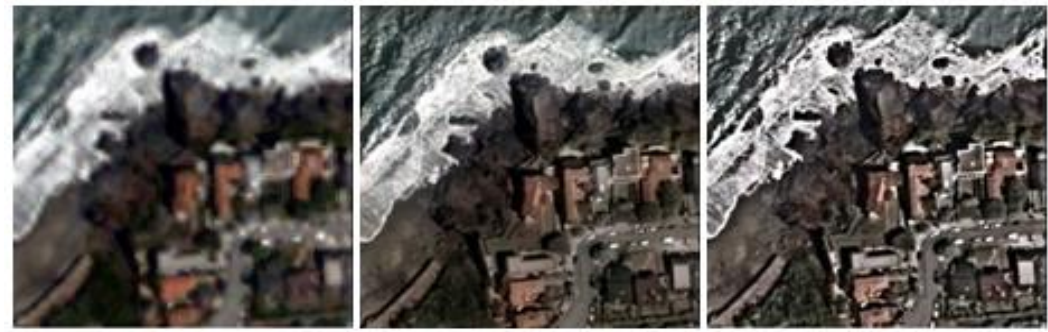

a) BEMD+Wavelet

b) BEMD+Curvelet c) proposed BEMD+Shearlet

Fig. 6. Comparison of different fusion methods by using Quickbird images 
JES, Assiut University, Faculty of Engineering, Vol. 44, No. 6, November 2016, pp. 727 - 737

Table 1.

Quantitative metrics of WorldView3 satellite images

\begin{tabular}{|c|c|c|c|c|c|c|c|}
\hline & \multicolumn{3}{|c|}{ Spat. } & Spec. & \multicolumn{2}{c|}{ (Spat. \& Spec.) Ave. } & All \\
\hline & MSE & Cor. & QNR & MSSIM & PSNR(DB) & ERGAS & UIQI \\
\hline BEMD+Wavelet & 1129 & 0.7817 & 0.48 & 0.9006 & 23 & 34 & 0.45 \\
\hline BEMD+Curvelet & 785 & 0.8289 & 0.575 & 0.9004 & 35 & 25 & 0.558 \\
\hline BEMD+Shearlet & 625 & 0.840 & 0.595 & 0.9005 & 37.5 & 23 & 0.6 \\
\hline
\end{tabular}

\section{Table 2.}

Quantitative metrics of Quickbird satellite images

\begin{tabular}{|c|c|c|c|c|c|c|c|}
\hline & \multicolumn{3}{|c|}{ Spat. } & Spec. & \multicolumn{2}{c|}{ (Spat. \& Spec.) Ave. } & All \\
\hline & MSE & Cor. & QNR & MSSIM & PSNR(DB) & ERGAS & UIQI \\
\hline BEMD+Wavelet & 1031 & 0.936 & 0.5596 & 0.90092 & 15.5 & 30 & 0.575 \\
\hline BEMD+Curvelet & 560 & 0.959 & 0.6093 & 0.9008 & 21 & 27 & 0.71 \\
\hline BEMD+Shearlet & 440 & 0.9628 & 0.6214 & 0.9009 & 22 & 26.5 & 0.735 \\
\hline
\end{tabular}
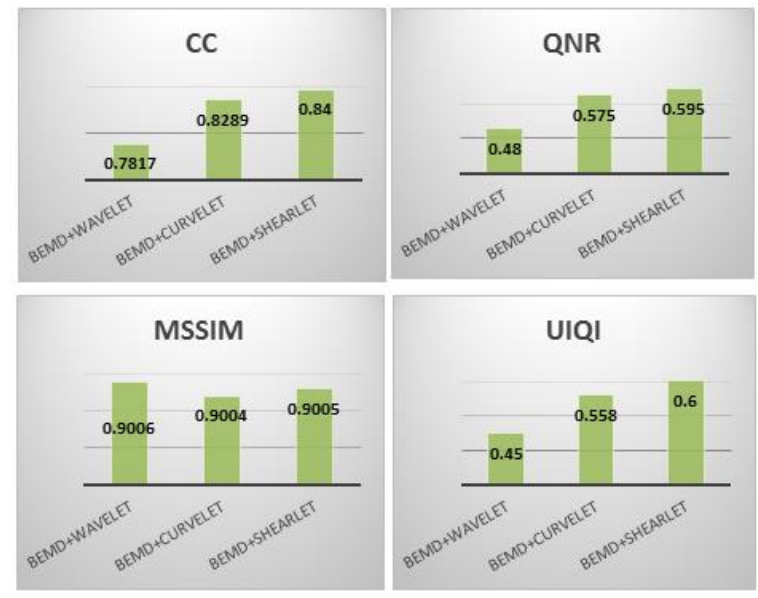

Fig. 7. Plots of quantitative metrics for WorldView3 images
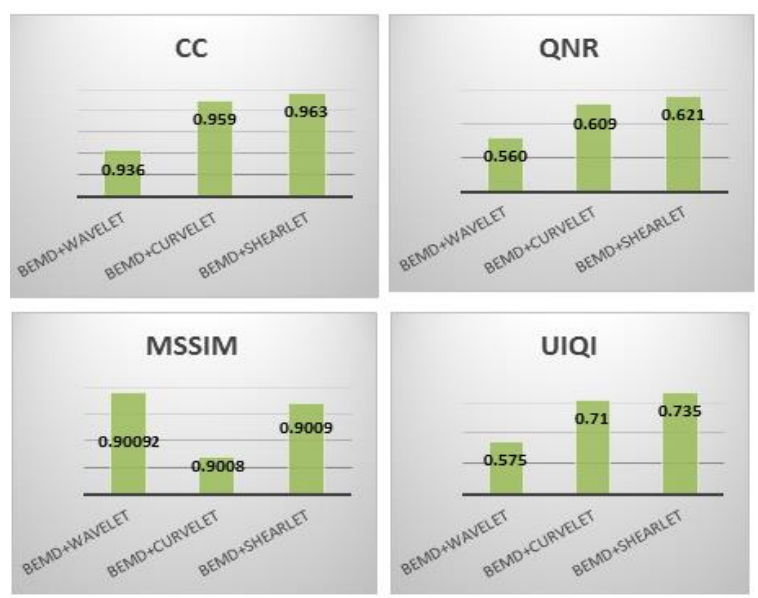

Fig. 8. Plots of quantitative metrics for Quickbird images. 


\section{Conclusion}

In this paper, a new method for enhanced fusion of MS and PAN satellite images has been proposed based on integrated scheme of BEMD and Shearlet transform. In addition, the automatic selection of fusion weights is optimized by implementing QGA. This new method has been tested with two datasets of WorldView3 satellite images; PAN image $(31 \mathrm{~cm}$ resolution) and a MS image (1.24 $\mathrm{m}$ resolution) and the other set of Quickbird satellite; PAN image (55 cm resolution) and a MS image (2.16 m resolution). Visual and quantitative metrics (MSE, Cor., QNR, MSSIM, PSNR, ERGAS and UIQI) are employed for spatial and spectral evaluations. Experiments confirm that the results generated by this new method are superior to results obtained using other illustrated methods. BEMD+Shearlet displays high performance in terms of maintaining spectral information of different land and sea cover types in different satellite images. On the other hand, it enhances the spatial details and increase clarity in edges and curves in satellite images. Experimental results demonstrated that proposed BEMD+Shearlet method appears to be a robust fusion method within different land cover images. At last, the effectiveness of proposed method on two different sensors images was proved. The method can obtain better image spatial details with less color spectral distortion.

\section{REFERENCES}

[1] Vivone G, Alparone L et al (2015) A Critical Comparison among Pansharpening Algorithms. IEEE Transactions on Geoscience and Remote Sensing 53(5):2565- 2586.

[2] Subramanian P, Alamelu NR, Aramudhan M (2015) Fusion of multispectral and panchromatic images and its quality assessment. ARPN Journal of Engineering and Applied Sciences 10(9):1849-6608.

[3] Do MN, Vetterli M (2002) Contourlets a directional multiresolution image representation. Proceedings of ICIP Rochester, New York, USA, pp 357-360.

[4] Labate D, Lim WQ, Kutyniok G, Weiss G (2005) Sparse multidimensional representation using shearlets. SPIE Proc. 5914, Bellingham, pp 254-262.

[5] Lim WQ (2011) Discrete shearlet transform faithful digitization concept and its applications. Proc. SPIE 8138, Wavelets and Sparsity XIV, 813810; doi:10.1117/12.893105.

[6] Huang NE, Shen Z et al (1998) The empirical mode decomposition and the Hilbert spectrum for nonlinear and non-stationary time series analysis. Royal Society of London Proceedings Series A, 454, pp 903-995.

[7] Nunes JC, Bouaoune Y et al (2003) Image analysis by bidimensional empirical mode decomposition. Image and Vision Computing, 21, pp 1019-1026.

[8] Looney D, Mandic DP (2009) Multiscale image fusion using complex extensions of EMD. IEEE Transactions on Signal Processing, 57(4):1626-1630.

[9] Demir B, Ertürk S (2008) Empirical mode decomposition pre-process for higher accuracy hyperspectral image classification. In Proceedings of IEEE International Geoscience \& Remote Sensing Symposium IGARSS, 2, pp 939-941.

[10] Ahmed MU, Mandic DP et al (2010) Image fusion based on Fast and Adaptive Bidimensional Empirical Mode Decomposition. In Proceedings of the IEEE 13th International Conference on Information Fusion, Edinburgh, UK, pp 1-6.

[11] Weihua D, Xian'en L et al (2014) A Bidimensional Empirical Mode Decomposition Method for Fusion of Multispectral and Panchromatic Remote Sensing Images. Remote Sensing 2014, 6(9):8446-8467.

[12] Narayanan A, Moore M (1996) Quantum-inspired genetic algorithms. Proceedings of IEEE International Conference on Evolutionary Computation, pp 61-66. 
JES, Assiut University, Faculty of Engineering, Vol. 44, No. 6, November 2016, pp. 727 - 737

[13] Huaixiao W, Jianyong L et al (2013) the Improvement of Quantum Genetic Algorithm and Its Application on Function Optimization. Mathematical Problems in Engineering, vol. 2013, Article ID 730749.

[14] Ben Arfia F, Ben Messaoud M et al (2012) Image Denoising in Gaussian and Impulsive Noise Based on Block Bidimensional Empirical Mode Decomposition. International Journal of Computer Applications (0975 - 888), 48(10):41-46.

[15] (2002) 2D empirical mode decomposition in the spirit of image compression. Wavelet and ICA Applications IX, in Proceedings of SPIE, Orlando, vol. 4738, pp 1-8.

[16] Hongzhi W, Yan L et al (2013) An Image Fusion Algorithm Based on Shearlet. Third International Conference on Information Science and Technology (ICIST); Yangzhou, China, pp 1625-1628.

[17] Easley G, Labate D et al (2008) Sparse Directional Image Representations using the Discrete Shearlet Transform. Applied Computational Harmonic Analysis, 25(1):25-46.

[18] Biswajit B, Abhishek D et al (2015) Remote Sensing Image Fusion using Hausdorff Fractal Dimension in Shearlet Domain. International Conference on Advances in Computing, Communications and Informatics (ICACCI), pp 2180-2185.

[19] Han K (2000) Genetic Quantum Algorithm and Its Application to Combinatorial Optimization Problem. In Proceedings of IEEE Congress on Evolutionary Computation, USA, vol. 2, pp 1354-1360.

[20] Jiang Sh, Zhou Q et al (2012) Analysis on parameters in an improved quantum genetic algorithm. International Journal of Digital Content Technology and its Applications (JDCTA), 6(18):176-184.

[21] Zhang G, Li N et al (2004) A novel quantum genetic algorithm and its application. Acta Electronica Sinica, 32(3): 476-479.

[22] Han K (2000) Genetic Quantum Algorithm and its Application to Combinatorial Optimization problem. In Proceedings of IEEE Congress on Evolutionary Computation, USA, vol. 2, pp. 1354-1360.

[23] Pandit VR, Bhiwani RJ (2015) Image Fusion in Remote Sensing Applications: A Review. International Journal of Computer Applications (0975 - 8887), 120 (10):22-32.

[24] Wang Z, Simoncelli EP et al (2003) multi-scale structural similarity for image quality assessment. Proceedings of the 37th IEEE Asilomar Conference on Signals, Systems and Computers, Pacific Grove, CA, vol. 2, pp 1398-1402. 


\section{خوارزمة دمج صور الاقمار الصناعية المحسنة باستخدام \\ التحليل التجريبى الثنائى الابعاد وتحويل ال Shearlet مبنى على الل بGA}

$$
\text { الملخص العربى }
$$

وان دمج الصور المتعددة الأطياف هو عملية دمج البيانات ذات الصلة من الصور الاحادية اللون (PAN)

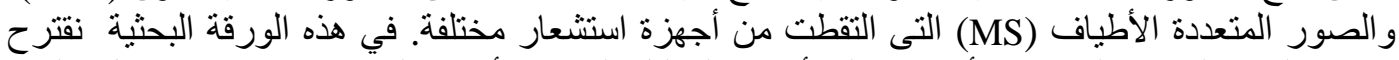

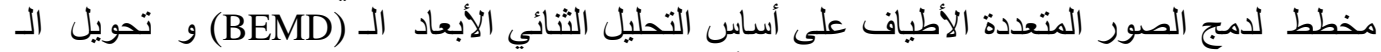
في BEMD يعتبر أداة قوية لتحليل الصيور متعددة النطاقات ، وميزته الرئيسية

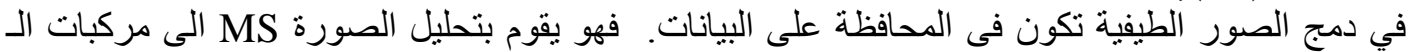

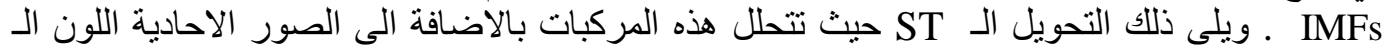

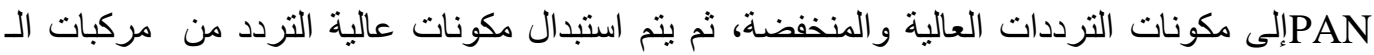
بمثيلاتها العالية التردد الخاصة بالصور الـ IMFs العكسية للتحليل الـ BEMD وكذللك التحويل الـ ST ST الحصول على الصورة المدمجة النهائية المتعددة

ان التحويل الـ ST يمكن أن يحقق التمثيل الأمثل للعناصر المتباينة الخو اص في الصورة بالاضافة انه

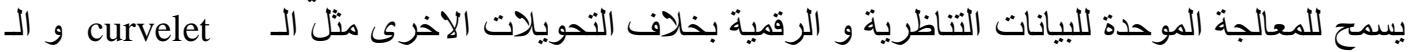
contourlet

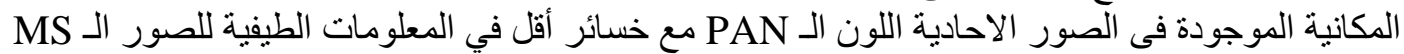

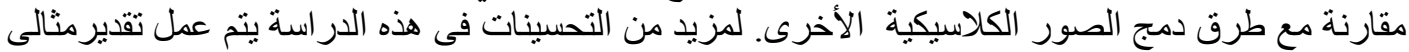

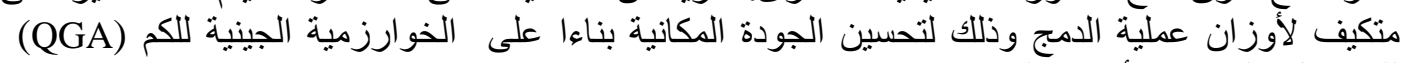
للحصول على هذه الأوزان تلقائيا. 